\title{
RACIONALIDADES E INTERESES DE CIENTISTAS SOCIALES PARA REALIZAR UN MAGÍSTER EN EL EXTRANJERO: IMPLICANCIAS PARA EL PROGRAMA BECAS CHILE
}

\section{El estudio}

El artículo se enmarca en un estudio que analiza el fenómeno de expansión de la educación superior y el auge de los posgrados en Chile, contrastando los supuestos discursivos y teóricos de la política pública de fomento de estos, particularmente de Becas Chile, con la perspectiva de quienes están accediendo a ellos, específicamente, de cientistas sociales que realizan un magíster en el extranjero.

En este marco, el objetivo principal del estudio fue comprender los intereses de cientistas sociales por hacer un magíster en el extranjero, para ello se abordaron tres objetivos específicos: indagar los aportes de un magíster en el extranjero, relevados por cientistas sociales; describir sus intereses por seguir estos estudios; e identificar racionalidades y lógicas presentes en el interés de estos profesionales por participar en este tipo de iniciativas.

Se utilizó una metodología exploratoria-descriptiva y cualitativa, basada en la revisión bibliográfica y la realización de entrevistas en profundidad semiestructuradas. La muestra se conformó por personas que al momento de la realización de la entrevista estuvieran postulando a Becas Chile para hacer un magíster en el extranjero en el área de las ciencias sociales (postulantes); y también por personas del área que ya lo habían terminado gracias a la asignación de la Beca Presidente de la República, entre 2000 y 2007, y que ya llevaban al menos seis meses desde su regreso (posgraduados).

Como se observa en la Tabla 1, en total se realizaron catorce entrevistas, ocho a posgraduados y seis a postulantes, los cuales se contactaron mediante la técnica de bola de nieve, la que consiste 
en identificar sujetos a incluir en la muestra a partir de los propios entrevistados, de modo que, partiendo de una pequeña cantidad de individuos que cumplen con los requisitos necesarios, se localizan a otros con características análogas. Cabe decir que la mayoría de los entrevistados quería estudiar o había estudiado en Inglaterra, lo que es consistente con el hecho de que la mayoría de las Becas Chile de magíster se destina a un posgrado en dicho país ${ }^{2}$. Además, la muestra final resultó estar compuesta equitativamente por una mitad de hombres y otra de mujeres.

Tabla 1: Composición y tamaño muestral

\begin{tabular}{c|c|c|c|c|c}
\hline $\begin{array}{c}\text { Tipo de } \\
\text { posgrado }\end{array}$ & Área & $\begin{array}{c}\text { Postulantes/ } \\
\text { posgraduados }\end{array}$ & País & Sexo & $N^{0}$ \\
\hline \multirow{2}{*}{$\begin{array}{c}\text { Magíster al } \\
\text { extranjero (con } \\
\text { beca estatal) }\end{array}$} & $\begin{array}{c}\text { Ciencias } \\
\text { sociales }\end{array}$ & Postulantes & $\begin{array}{c}\text { Inglaterra, Holanda, } \\
\text { Australia, Argentina, Brasil }\end{array}$ & $\begin{array}{c}3 \text { mujeres y } \\
3 \text { hombres }\end{array}$ & 6 \\
\cline { 3 - 6 } & & Posgraduados & Inglaterra, Francia & $\begin{array}{c}4 \text { mujeres y } \\
4 \text { hombres }\end{array}$ & 8 \\
\cline { 3 - 6 } & & & & 14 \\
\hline
\end{tabular}

El principal criterio para seleccionar a personas que querían realizar o habían realizado un magíster se refiere a que, a diferencia de lo que suele ocurrir con los doctorados, este tiende a constituirse como una primera instancia de posgrado, esto propicia una frescura en las significaciones de los entrevistados respecto de los porqués para seguir estudiando. Por su parte, y como se verá más adelante, el motivo fundamental para que estos posgrados fueran en el extranjero y con beca estatal es consecuente con el diagnóstico inicial de este estudio: la existencia de un discurso público que promueve la educación de posgrado, donde las recientes políticas de aumento de becas serían su mayor expresión, siendo estas mayormente becas para estudiar en el extranjero (Becas Chile).

Para mayor información se puede consultar el siguiente enlace: http://www.becaschile.cl/ index2.php?id_seccion=4003\&id_portal=60\&id_contenido=17946 
Por otro lado, la elección de profesionales de las ciencias sociales $^{3}$ se realizó considerando que es el área disciplinaria que concentra una mayor cantidad de becas de magíster para el extranjero, con poco menos de la mitad de ellas hasta 2012 (49,1\%) (Becas Chile, $2012)^{4}$. Finalmente, la decisión de entrevistar tanto a postulantes como a posgraduados surge de un interés por enriquecer el estudio al indagar en las dos facetas (motivación y evaluación) de los principales involucrados en el acceso a los posgrados en cuestión.

Es necesario clarificar que no fue pretensión de esta investigación alcanzar representatividad estadística, ya que su validez como estudio cualitativo no se funda en un criterio extensivo, sino que en uno intensivo: no se buscó extender de manera universal a una población mayor una información extraída a partir de un estudio de casos concretos, sino que se priorizó la profundidad de la información.

La información producida se transcribió, sistematizó y analizó a partir de la estrategia de análisis de contenido, con el fin de identificar y examinar los significados y sentidos presentes en las entrevistas, desde una perspectiva emic que privilegia un acercamiento a las categorías del otro (Tudela, 2006).

Dicho análisis se realizó a la luz de una complejización de los conceptos de "interés" y "racionalidades" (Bourdieu, 1991, 1997, 2003), que permite discutir la capacidad explicativa del discurso público de fomento al posgrado y la teoría del capital humano a la base de esta política para comprender, por sí solos y bajo la asunción de un sujeto racional instrumental, las lógicas de interés que se ponen en juego en la realización de posgrados. Como se verá, los resultados evidencian la existencia de, al menos, dos racionalidades que motivan la realización de estos estudios: una estratégica, orientada al posicionamiento laboral y otra experiencial, orientada a la individuación. Este hallazgo puede implicar importantes

3 Esta área se definió a partir de las categorizaciones disciplinarias definidas por la Organización para la Cooperación y Desarrollo Económico (OCDE), utilizadas por Conicyt. Estas son: Psicología, Economía y Negocios, Ciencias de la Educación, Sociología, Leyes, Antropología (Social y Cultural), Etnología, Demografía, Geografía Social y Económica, Ciencias Políticas, Métodos y Organización, Periodismo y Comunicaciones, y disciplinas afines.

4 Los datos para 2013 no se encuentran disponibles a mayo de 2014. 
consecuencias para la política pública de becas de posgrado en el extranjero.

\section{Contexto y problematización}

\subsection{Expansión del pregrado y auge del posgrado: los cambios}

Tras la Segunda Guerra Mundial ciertos procesos comenzaron a potenciarse de manera global, dando forma a lo que hoy se denomina "sociedad del conocimiento". Dichos procesos implican la reorganización de las esferas de la vida política, social y económica de los países, entre ellos Chile, y con ello la emergencia de nuevas formas de producción y de transferencia de tecnologías y conocimientos. En este contexto, la educación adquiere una importancia central, porque sus tareas se consideran como directamente relacionadas con los niveles de crecimiento económico, desarrollo social y calidad de vida de los países, así como también la base para la competitividad y la productividad nacional. Es por esto que hay una preocupación por los denominados "recursos" o "capitales humanos", es decir, por las habilidades y competencias de las personas para contribuir al desarrollo del país, y ya no solo una preocupación por los recursos económicos, sean estos naturales, productivos, etc. (Didriksson, 2008).

En este marco se entiende la expansión de la cobertura educacional en Chile y, dentro de este proceso, la creciente masificación de la educación superior de pregrado: en poco más de 50 años la matrícula aumentó 45 veces, pasando de 20.000 alumnos matriculados en 1957 a 637.226 en 2005 y 900.936 en 2010 (CSE, 2011). Este proceso se inicia en los años sesenta, en concordancia con los movimientos de modernización que derivan en una mayor demanda de mano de obra calificada por parte del Estado. Consistentemente con esto, desde 1967 la expansión del pregrado se intensifica gracias a la duplicación del financiamiento público. No obstante, esta alza cambia de rumbo en 1974 cuando, con la dictadura militar, el gasto público en educación se reduce bruscamente, llegando a disminuir en $35 \%$ en 1981, producto de 
las reformas de privatización. El Estado asume un rol subsidiario en el financiamiento educativo, permitiendo que el mercado controle la oferta (instituciones y carreras que se ofrecen) y la demanda (posibles alumnos) educacionales, instando así a que las universidades se desarrollen utilizando otras fuentes de financiamiento. A partir de esto se fomenta la creación de universidades privadas y nuevas instituciones no universitarias de educación superior, como son los Institutos Profesionales (IP) y los Centros de Formación Técnica (CFT) (CNAP, 2007).

De este modo, las políticas implementadas en la década de los años ochenta marcan el inicio de procesos de privatización y mercantilización que constituirán al sistema de educación superior en un mercado cuyas características principales son la ausencia de cualquier regulación externa, la competencia y la liberalización de la oferta y de la demanda (Brunner y Uribe, 2007). La conformación de este mercado tiene como consecuencia directa la masificación y expansión del sistema de educación superior, procesos que no se detienen al terminar la dictadura, sino que más bien se consolidan, independientemente de que el gasto público en educación durante los gobiernos de la Concertación aumentó en 140\% entre 1990 y 2006. En este proceso, la expansión de la educación superior de posgrado es indisociable de la creciente masificación de la de pregrado. Pese a que la cantidad de chilenos con posgrado es aún pequeña, llegando a 0,8\% de la población en 2009 (CASEN, 2009), esta ha ido aumentando muy notoriamente en los últimos años. Así, entre 1992 y 2009 la cantidad de chilenos con posgrado aumentó en 547,8\% (CASEN, 1992-2009).

Sin duda aquí las becas juegan un papel central y determinante de este nuevo contexto de fomento institucional al posgrado. Esto resulta muy claro para el caso de las becas de posgrado para estudiar en el extranjero, en un inicio mayormente concentradas en las Becas Presidente de la República -administradas por el Ministerio de Planificación, Mideplan- y desde 2009 centralizadas en Becas Chile -administradas por la Comisión Nacional de Investigación Científica y Tecnológica,Conicyt-. Se observa un aumento de la cantidad de becarios a inicios de 2000, lo que se vuelve muy marcado a finales del decenio con la creación de Becas Chile, fondo público establecido 
en 2008 que se proyectó con 6.000 millones de dólares a invertir, en el lapso de una década, exclusivamente en becas de estudio en el extranjero. Con dicha inversión se previó que la cantidad de becados para el extranjero llegaría a 5.000, cifra que iría in crescendo para que a contar de 2017 hubiera 30.000 chilenos con posgrados extranjeros trabajando en el país. Este es un esfuerzo doce veces mayor al realizado en los últimos 20 años y que pone de manifiesto una cuantiosa inyección de recursos por parte del Estado chileno, relevando la importancia de aumentar el nivel educacional de la población: si desde 1981 a 2008 la Beca Presidente de la República envió a 2.787 becados al extranjero, Becas Chile asignó, entre 2009 y 2010, es decir, en solo dos años, casi la misma cantidad (2.379 becas) (Márquez y Heyl, 2010; Statcom Estadística, 2007).

Estas iniciativas no son exclusivas de Chile, sino que se trata más bien de una tendencia internacional a la que el país se integró. En efecto, China, México y Brasil son algunos de los países clave en esto. Sin embargo, resulta llamativo el que Chile sea uno de los países que mayor inversión estatal ha contemplado. Por dar un ejemplo, el gobierno chino se ha concentrado en facilitar la partida de los estudiantes -lo que no necesariamente se traduce en su financiamiento- dejando la mayor parte de los costos de los estudios al autofinanciamiento (OCDE-Banco Mundial, 2011), con esto se reafirma la amplitud e intensidad con que se proyectó la política chilena de fomento al posgrado.

Así, si bien ya a mediados de la década pasada la cantidad de becas para estudiar en el extranjero iba en aumento, llegando a 310 el 2007 (Universia, 2007), esta suma se incrementa radicalmente los primeros años de implementación de Becas Chile, superando las 1.300, entre magíster y doctorado en el 2009. Ahora bien, la expansión del posgrado puede observarse como un proceso continuo mas no lineal: con la llegada del nuevo gobierno, en 2010 se redujo el presupuesto para este programa, entregándose solo 775 becas, entre magíster y doctorado. Esta cantidad de becas se mantuvo estable durante los cuatro años de gobierno de Sebastián Piñera. En concordancia con esta tendencia, se realizaron críticas y reformas a Becas Chile, aludiendo principalmente a un exceso de 
recursos invertidos en comparación con los retornos sociales que ello implicará.

Con esto se evidencia el carácter aún emergente de una política de educación avanzada y continua a nivel nacional. En este contexto, la expansión del posgrado merece ser atendida en profundidad y en sus distintas aristas. Una de ellas son los elementos conceptuales que están en los fundamentos de esta política pública.

\subsection{El discurso público de fomento al posgrado}

En su página web, el programa Becas Chile señala como objetivo fundamental:

(...) la definición de una política integral de largo plazo para la formación de capital humano avanzado en el extranjero. Lo que promueve a través de tres líneas de acción: a) Aumentar las oportunidades de formación y perfeccionamiento en el extranjero; b) Modernizar y articular los programas de becas gubernamentales; y c) Fomentar la vinculación y cooperación internacional (Becas Chile, 2009).

Considerando estos fines, así como el Decreto Supremo Nº66 que da origen al programa (Mineduc, 2009), y el análisis hecho por el estudio Evaluación en profundidad de los programas de becas de posgrados (Statcom Estadística, 2007) -que generó recomendaciones a los programas de becas de posgrado, entre ellas las becas para estudios en el extranjero (Beca Presidente de la República)-, fue posible identificar ejes clave de la política pública en torno a estas becas, en su articulación con determinados planteamientos teóricos: igualdad de oportunidades, meritocracia y movilidad social.

Un primer aspecto por destacar es que las facilidades económicas para hacer un posgrado, entre ellas las becas, se conceptualizan como elementos necesarios para la constitución de una sociedad basada en la igualdad de oportunidades: se entienden como uno de los mecanismos existentes para suprimir o compensar las desigualdades existentes en relación con el acceso a oportunidades asociadas a la educación. De este modo, el discurso de fomento al posgrado por medio de becas buscaría, en sus fundamentos, posibilitar que ningún talento se pierda 
(Emol, 2008), por motivo de esas desigualdades iniciales. Siguiendo la definición de Atria (2004), esto concuerda con el desarrollo de una sociedad equitativa en la medida en que este tipo de política busca no solo respetar la igualdad de las personas ante la ley, sino también promover la eliminación de las barreras socioeconómicas y sociales con el fin de lograr la realización del potencial individual.

Junto con este principio de equidad, esta política es consistente con un modelo de sociedad alentado por el ideal de la meritocracia, donde las posiciones sociales sean conseguidas sobre la base del esfuerzo, talento y méritos individuales, y no a partir de características hereditarias o adscritas, como el sexo o la riqueza de cuna. En este sentido, asumiendo una plataforma de condiciones equitativas para acceder a las oportunidades sociales sería posible que las diferencias entre las personas se generen únicamente considerando sus méritos y logros, los cuales se miden principalmente a partir de su desempeño educacional. Es decir, asegurando la equidad en las oportunidades sería posible y válido que se produzcan desigualdades posteriormente, ello mediante mecanismos objetivos y transparentes de selección y distribución de roles, como serían las evaluaciones (por ejemplo, la Prueba de Selección Universitaria, PSU).

Esta forma de diferenciación, al contrario de aquella transmitida por herencia, estaría legitimada en tanto se sustenta en una base de igualdad. Así, serán los más esforzados quienes alcancen niveles avanzados de educación y, por lo tanto, se entiende que la posición que cada cual ocupa en la estructura social es la que cada cual se merece de acuerdo con su desempeño. Se trata de una sociedad basada en el logro antes que en la adscripción (Crompton, 1994), algo que hace medio siglo el sociólogo funcionalista Talcott Parsons (1959) ya había defendido como un objetivo de la sociedad moderna.

Debido a estas condiciones, el origen social de los individuos no se configura como un aspecto determinante de las posiciones, oportunidades y condiciones de vida por alcanzar. Una sociedad equitativa y meritocrática es, a la vez, una sociedad con altos niveles de movilidad social en la que las posiciones de los sujetos no son estáticas y, por lo tanto, pueden experimentar cambios. La movilidad 
social constituye tal vez la mayor promesa de la sociedad neoliberal actual, la de que cada cual puede ser lo que quiera ser.

\section{3. ¿La educación como inversión?}

Los ejes antes mencionados engarzan con la denominada teoría del capital humano, la que asume que la educación es una herramienta que entrega las habilidades y conocimientos para desempeñarse productivamente en el trabajo, desempeño que será más productivo mientras mayor sea el nivel educacional con el que se cuente. De aquí que mientras más personas tengan altos niveles educacionales $\mathrm{y}$, por tanto, sean más productivas en su trabajo, mayores serán los niveles de productividad, crecimiento y desarrollo económico nacionales (Dridriksson, 2008; Marginson, 2008; Patrón, 2008; Brunner y Uribe, 2007; Spencer, 2003; Franco, 2002; Rodríguez y Vio, 2003; CEPAL, 2000). A partir de esto se entiende que los estímulos del gobierno para la realización de posgrados forman parte de los esfuerzos por apoyar una economía basada en el conocimiento, en pos de ser un país competitivo internacionalmente. De aquí la centralidad de fomentar la realización de posgrados en el extranjero.

Pieza clave en este mecanismo serían las recompensas laborales al aumento en la capacidad productiva, por medio de mayores ingresos y mejores condiciones laborales; recompensas que, al ser identificadas por los sujetos, motivarían su decisión de estudiar, de manera que las ganancias individuales se convierten en un incentivo para las ganancias nacionales. A partir de esto se entiende que los sujetos reconocen la decisión de estudiar como una inversión -tanto en términos monetarios como temporales-, pues buscan principalmente incrementar sus habilidades y conocimientos para así aumentar su eficiencia productiva y, a partir de esto, sus ingresos (Schultz, 1960; Becker, 1983, 1990; Destinobles, 2006; Rodríguez-Sickert, 2009).

Así, esta decisión resulta de un principio de economía y de un cálculo maximizador que considera, por una parte, los costos presentes -expresados en el costo de oportunidad de dejar de realizar otra actividad rentable, y el costo directo de los estudios como por ejemplo el pago de la matrícula, traslados, materiales, 
etc.-; por otra parte considera los beneficios o retornos futuros esperados -expresados en mayores salarios y no monetariamente, por ejemplo, en la satisfacción derivada del trabajo-. En este esquema, los beneficios futuros -en especial los monetarios- deben ser mayores que los costos presentes (Becker, 1983). A esto se suma que se trata de una decisión libre, es decir, que depende del individuo (Mincer, 1958; Montuschi, 2006), y que por lo tanto puede ser controlada por este. Esto es consistente con una concepción del ser humano como homo economicus, muy anclada en la teoría de la acción racional predominante en la ciencia económica, basada en la primacía de la racionalidad instrumental.

Ahora bien, la Antropología Social y la Sociología han puesto en tela de juicio la preeminencia de la racionalidad instrumental para entender por qué las personas hacen lo que hacen. Antropólogos como Karl Polanyi (1989), Marcel Mauss (1971), Marshall Sahlins (1983), Maurice Godelier (1966) y Claude Lévi-Strauss (1964) han demostrado las homologías entre el pensamiento del hombre moderno y el del mal llamado "salvaje", así como el que en las sociedades tradicionales las elecciones que hace la gente en materia de producción e intercambio de bienes siguen patrones que difieren considerablemente de lo que el modelo del homo economicus postula. Asimismo, habrían sociedades que no se orientan sobre la base de las reglas de la utilidad monetaria, pese a conocerlas (Godelier, 1966). De este modo, el principio del cálculo y la maximización constituyen un universo dentro de los muchos que existen en la economía. Hay sociedades que no se orientan por él (Sahlins, 1983), por lo que no es un interés necesario sino contingente y vinculado a características sociales particulares. En este marco, se entiende que los sujetos tengan intereses variados y no necesariamente utilitarios y lucrativos, de manera que el valor que los objetos y/o fenómenos educativos tiene para ellos no es posible de entender solo a partir de la instrumentalidad. Desde esta perspectiva, acciones en apariencia desinteresadas pueden estudiarse en términos de interés.

En esta perspectiva, el sociólogo Pierre Bourdieu esboza una Antropología Económica que alude al carácter reduccionista del "imperialismo" de la racionalidad instrumental para entender 
los intereses de los sujetos. Sus razones serían reducidas a razones conscientes y calculadas, como si estos se plantearan deliberadamente los fines de su acción y actuaran para conseguir la máxima eficacia al menor coste. Sin embargo, esto sería más un supuesto y una proyección de los investigadores que un principio de acción de los sujetos: el hecho de que estos no hagan cualquier cosa y que no actúen de manera arbitraria y sin razón -"a tontas y a locas"- no quiere decir que se rijan por dichos principios. A partir de esto se propone hablar de acciones "razonables" más que "racionales", aludiendo a comportamientos de los que se pueda dar razón sin que estos hayan sido regidos, a cabalidad, de manera racional-instrumental (Bourdieu, 1991, 1997, 2003). Siguiendo dicho enfoque, este estudio entiende que las acciones y decisiones son "razonables" antes que "racionales", y que por lo tanto pueden involucrar distintas racionalidades de acción, más allá de la racionalidad instrumental usualmente esgrimida en la disciplina económica, así como en la política pública, a la luz de la teoría de la acción racional.

\section{Discusión y resultados}

Sobre la base de estas nociones y a la luz de la información producida, se observa una insuficiencia del concepto de racionalidad instrumental para entender los intereses de cientistas sociales por hacer un magíster en el extranjero, sino que más bien se identifican dos racionalidades intervinientes: una estratégica y otra experiencial.

\subsection{Racionalidad estratégica}

Es similar a la instrumental, pero siguiendo la conceptualización que hace el sociólogo francés Pierre Bourdieu del concepto de estrategia, permite entender que los sujetos inviertan en educación, poniendo en juego intereses y haciendo uso de sus oportunidades, sin que necesariamente se guíen por los principios del cálculo, la maximización y el libre albedrío. En efecto, pese a no ser calculados de manera racional en sus consecuencias, los comportamientos pueden orientarse a la obtención de beneficios o ganancias futuras, de modo que pueden observarse en términos de estrategia e incluso de inversión: si bien los sujetos están condicionados por su situación, 
no responden mecánicamente a ella, sino que pueden sacar provecho de sus oportunidades. Los agentes desarrollan estrategias aunque muy pocas veces estas realmente se fundamenten en una verdadera intención estratégica (Bourdieu, 1991, 1997, 2003).

Esta racionalidad estratégica se caracteriza por la valoración del magíster como ente propiciador de un posicionamiento laboral favorable, el que se define principalmente por cuatro intereses o lógicas de interés:

a) En primer lugar, los entrevistados observan que el magíster favorecerá un piso más alto de ingreso en el ámbito académico, aunque no mucho más (como sucedería en una consultora), que articula lo que aquí se ha denominado como una "lógica monetarista". En efecto, se observa que los entrevistados piensan que el magíster permite acceder a sueldos mayores, tanto porque el mercado laboral reconoce esta situación y la recompensa como porque los mismos profesionales se sienten con más derecho y autoridad para cobrar más. Así lo señalan postulantes y posgraduados:

Mi mamá ha trabajado en varias cosas y me cuenta, me dice que el magíster es fundamental, que incluso en sueldo son, no sé, 200 mil pesos más, aunque hagai lo mismo que el otro, por el solo hecho de tener el magíster (Hombre postulante).

Y hay una cuestión de las lucas también. Si antes de irme yo cobraba "A" por hora, ahora cobro "C" por hora, entonces también eso me permite a mí estar más contenta con mi vida, no tener que aperrar hasta las 11 de la noche todos los días. (...) Te sientes con derecho a cobrar por entregar el conocimiento que tienes (Mujer posgraduada).

b) En segundo lugar, hay una creencia de que poseer el magíster aumentará la libertad para elegir trabajos según preferencias y gustos personales, así como confianza en las capacidades laborales, la que se asocia a una "lógica de la autonomía" y de control dentro del mercado laboral. Por ejemplo, una mujer que hizo un posgrado en el extranjero señala: 
Hay un tema de autoestima o de percepción de uno mismo como profesional que te ayuda a pararte. Y es clave, si tú tení confianza en que podí hacer algo, te relacionai mejor, eres capaz de plantear las cosas en forma más relajada. Te puedes sentar a armar un proyecto con más calma. Es súper básico. Cuando uno está inseguro con uno mismo y deci "ipero qué cacho yo?", que era mi actitud antes de irme, "pero esto no lo manejo, no sé qué, ¿cómo se hace?” (...) En cambio después de estar allá [afuera, haciendo el magíster], de aperrar, decí "yo puedo inventarme, puedo aprender esto", o "tengo un par de ideas que... bueno, si me pescan bien; y si no me pescan, bien también". Menos miedo a equivocarse, más seguridad (Mujer posgraduada).

c) La posibilidad de distinguirse frente a otros profesionales que no tienen posgrado, o bien, que tienen posgrados peor evaluados que los que se poseen, vinculada a una "lógica de la distinción". Aquí son cruciales las características del posgrado, como por ejemplo el país de realización y la universidad, ya que por ejemplo:

En la universidad (...) éramos tres los que habíamos hecho magíster fuera. Dos tenían doctorado. Entonces claro, igual marcaba una cierta diferencia (Hombre posgraduado).

d) En cuarto lugar, hay un interés por mejorar la inserción y mantención en trabajos académicos, que se asocia a una "lógica de la necesidad", en tanto aquí el magíster viene a verse como un requisito mínimo de entrada a dicho ámbito. En este sentido, hay un reconocimiento de la insuficiencia del pregrado para cumplir esta función -pese a que se cuenten con la capacidades necesariasy, con ello, la creencia de que el magíster supla esta condición. La cita a continuación es clarificadora de dicho reconocimiento:

No teniendo magíster no podría hacer muchas cosas. No podría empezar a proponer muchas cosas. Quizás no estaría tan validada en muchas cosas. No podría estar a cargo de la Dirección de Investigación, por ejemplo. No lo podría hacer por validación; por capacidad quizás sí (Mujer posgraduada).

Junto con estos intereses, en esta racionalidad aparecen como especialmente relevantes dos tipos de aportes del magíster: 
el capital cultural o aprendizajes y el capital simbólico o estatus (Bourdieu, 1991), siendo el segundo especialmente relevante en tanto los aprendizajes se tornan determinantes en la estrategia de posicionamiento laboral a partir de su simbolización. Esto equivale a decir que más allá de que se aprendan ciertas habilidades, estas pasan a ser relevantes para acceder al mercado laboral si es que están credencializadas (Collins, 1989), y no solo eso: credencializadas de un determinado modo, por ejemplo, por una universidad prestigiosa en un país prestigioso.

Estos hallazgos, sintetizados en la Figura 1, sugieren que los sujetos están reconociendo la exigencia cada vez mayor de niveles educativos por parte del mercado laboral, muy posiblemente por la saturación que experimenta la educación superior de pregrado ante su proceso de masificación (Passeron, 1983).

Figura 1: Componentes de la racionalidad estratégica

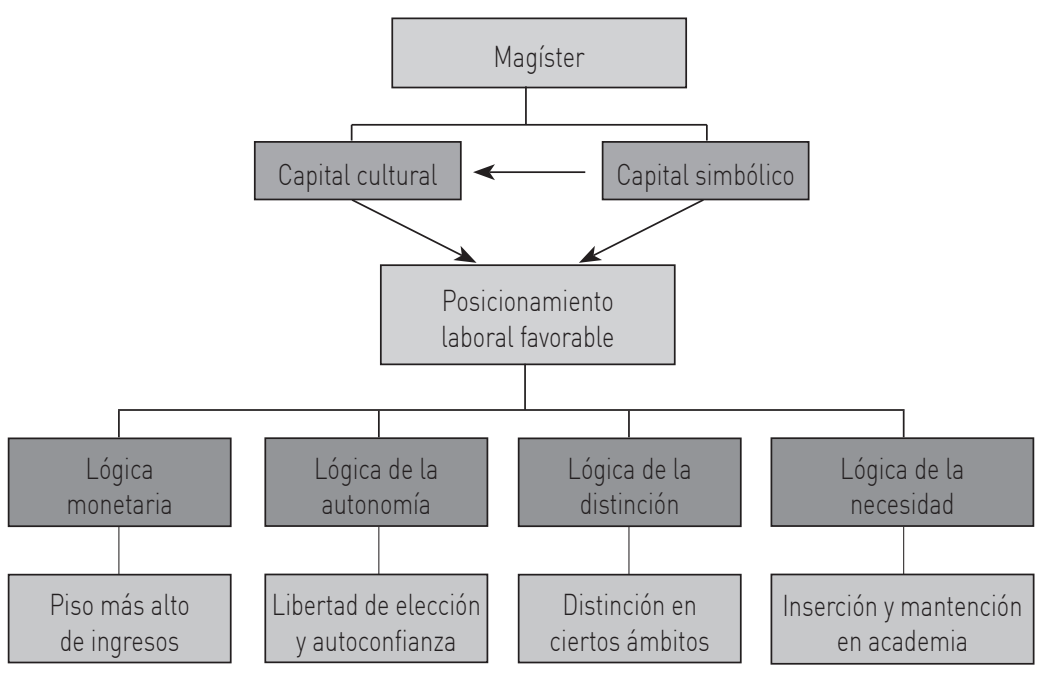

\subsection{Racionalidad experiencial}

Por otra parte, se identifica una racionalidad experiencial presente en el interés por hacer un magíster en el extranjero. Si bien la información producida es más escueta en relación con este aspecto -y por lo mismo sería interesante profundizarla en futuros estudios- 
se observa que además del posicionamiento laboral hay un gran aprecio por la experiencia misma de vivir en el extranjero como vía para la individuación. En este sentido, está operando una "lógica del crecimiento personal", apoyada en los siguientes aspectos:

a) En primer lugar, se observa un interés por el intercambio cultural inherente a la realización de estudios en el extranjero, en tanto permitiría interactuar con gente de costumbres distintas pero con intereses intelectuales similares. De este modo, las redes informales aparecen como claves en la definición de la experiencia. Tanto postulantes como posgraduados hacen referencia a este aspecto:

Igual allá teníamos una vida donde nuestro tiempo libre, aunque estábamos carreteando, siempre estábamos todos con ene temas en la cabeza, todos estábamos leyendo libros, súper metidos en la realidad internacional. Es pura gente con mucho interés intelectual, entre comillas, en sus áreas y todo pero todos súper motivados (...). Y nos pasa que muchos de nuestros amigos que se quedaron acá de lo único que hablan es de la guagua y el perro. Como que hay un vuelo de apertura de cabeza como pa' preocuparse del mundo que lo compartimos ene con nuestros amigos de allá (...). Esa es una ganancia súper importante de los estudios afuera (Mujer posgraduada).

En la medida en que conoce otras cosas, lee otras cosas, aprende, comparte con otros que han vivido otras experiencias, uno aprende (Mujer postulante).

b) En segundo lugar, se reconoce el valor de tener tiempo para dedicarse a estudiar y a profundizar en temas de interés. Esto es reiteradamente señalado por posgraduados, aunque en menor medida también por algunos postulantes:

El tema de viajar e ir a estudiar te permite estar años centrado en un tema que te interesa, aprender de otros lugares, vivir otras experiencias (Hombre posgraduado).

Cuando uno se va uno tiene toda la energía puesta en eso, en estudiar harto, en hacer bien lo que estai haciendo, en aprender lo que más podai, vai a todas las charlas, a todas las exposiciones. Como que tu tema es perfeccionarte, entonces estai súper concentrado (Mujer posgraduada). 
Siento que me haría bien estudiar (...) es mi actividad favorita (Mujer postulante).

c) Por último, se releva la importancia de "experimentarse" o ponerse a prueba en un contexto diferente. Así como el componente "extranjero" es determinante en términos estratégicos mediante, por ejemplo, la valoración del idioma, aquí este elemento destaca a partir de la apreciación del hecho de vivir afuera, en un lugar anhelado, entendido como un paréntesis en la rutina actual, muy marcado por el carácter de gratuidad (por la beca) de los magísteres en cuestión. Esto es algo señalado por postulantes de distintos sexos:

Me di cuenta que en verdad lo único que quería era irme a afuera un tiempo, que no quiero quedarme acá toda la vida. Tener esa experiencia (Hombre postulante).

Además del rollo académico para mí era importante elegir un buen lugar para vivir. O sea, por mucho que en Ciudad Juárez estuviera la mejor universidad en derechos humanos del universo no me iría a meter allá. No me iría. Como que igual... también calidad de vida, todo el rato (Mujer postulante).

Como se observa hay, por una parte, una valoración del capital cultural aportado por el magíster y, por otro, se reconoce la importancia de capitales culturales que trascienden a la instancia misma del posgrado y que dicen relación con las circunstancias en las que este se da, específicamente con el hecho de que se realice en otro país. De cualquier modo se trata, en última instancia, de una valorización personal del magíster por su capacidad de incorporarse y de ser reconocido socialmente como propiedad intrínseca (y no como producto de, por ejemplo, un privilegio social). Es por ello que puede decirse que el capital adquirido en el proceso educacional vale no solamente en el mercado del trabajo, sino que da valor a la persona en sí (Passeron, 1983).

Dicho esto, es posible pensar que la racionalidad experiencial se puede estar asociando con una valoración del magíster en el extranjero como soporte para la individuación y, en este sentido, como un elemento de construcción del proyecto personal, lo que siguiendo a 
Danilo Martuccelli (2007) implica que la experiencia es la base para la individuación, como proceso de fabricación de individuos:

(En la sociedad actual) se impone a los actores la necesidad de diversificar su individualidad, de fabricarse un currículum vitae interesante, tener éxito en sus estudios y en su profesión, hablar varias lenguas, vivir experiencias diversas, tener habilidades raras o seguir formaciones exclusivas; en breve, es necesario que el actor muestre que es alguien interesante para que los otros se interesen en él (Martuccelli, 2007, p. 104).

La Figura 2 sintetiza los aspectos constituyentes de la racionalidad experiencial, cuya presencia, que se articula con una racionalidad estratégica, es relevante en tanto expande la definición del posgrado desde uno caracterizado como proyecto laboral a uno sociopsicolaboral.

Figura 2: Componentes de la racionalidad experiencial
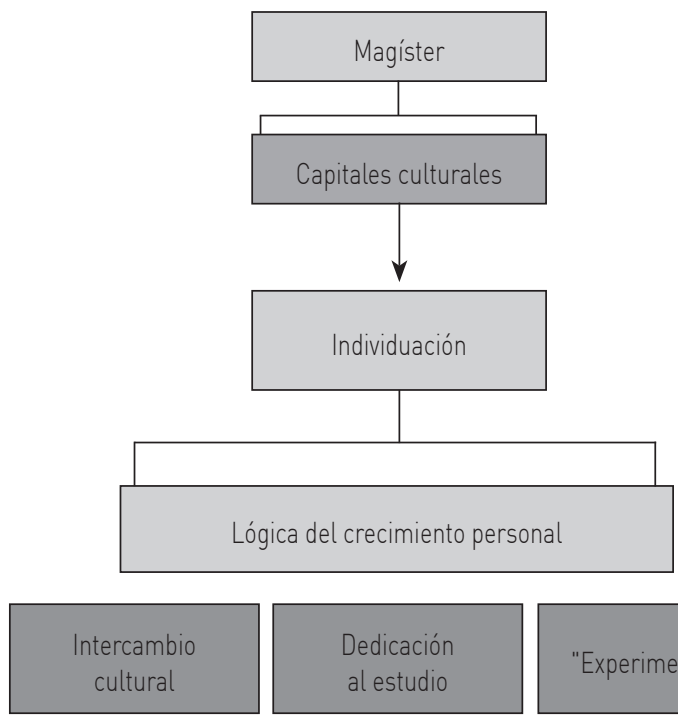

Dedicación

al estudio

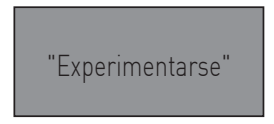

\section{Palabras finales}

Los hallazgos evidencian la insuficiencia del concepto de racionalidad instrumental contenido en la política pública de fomento de posgrados, por medio de la teoría del capital humano, para entender los intereses 
de cientistas sociales por hacer un magíster en el extranjero. Se identifican al menos dos racionalidades intervinientes: una estratégica y otra experiencial. La primera se caracteriza por la valoración del magíster por propiciar un posicionamiento laboral favorable, definido principalmente por cuatro lógicas de interés:

- lógica monetarista, con foco en favorecer un piso de ingresos más alto;

- lógica de la autonomía, con foco en incrementar la libertad de elección de trabajos;

- lógica de la distinción, con foco en diferenciarse de otros profesionales; y

- lógica de la necesidad, donde el magíster aparece como un requisito mínimo para insertarse y mantenerse en trabajos académicos.

Por su parte, la racionalidad experiencial se sostiene en un proyecto de individuación, apoyado en una lógica de crecimiento personal, muy marcada por el carácter internacional de estos posgrados, que valora el intercambio cultural, el tiempo para dedicar al estudio de temas de interés, y la posibilidad de "experimentarse" en un contexto diferente.

De este modo, cada racionalidad se define por distintas lógicas que, en su conjugación, delimitan distintos objetivos por perseguir. A su vez estos objetivos se asocian con los distintos aportes aparejados al posgrado: sus capitales culturales (aprendizajes) y simbólicos (estatus). La contribución estratégica del magíster sería fundamentalmente simbólica, de manera que los aprendizajes se tornan determinantes a partir de su simbolización. De la mano de esto se afirma que el magíster constituye una credencial, ya que permite incluir en el trabajo y en la sociedad -cuando el pregrado se comienza a saturar- un incipiente nuevo peldaño en el mecanismo de cierre social que es la educación. Por otra parte, la contribución experiencial se afirmaría principalmente en la adquisición de un capital cultural, definido por aprendizajes e intercambio con otros.

La presencia de la racionalidad experimental, en su articulación con la estratégica, es relevante en tanto expande la definición del 
posgrado desde una caracterizada en términos de proyecto laboral a una sociopsicolaboral.

A partir de estos resultados se hace necesario destacar que, pese a que la política pública de fomento al posgrado ha propiciado una contextualidad que los sujetos están aprovechando, ya que hay una demanda efectiva por las becas de posgrado al extranjero, no hay una correspondencia "uno a uno" entre los postulados de esta política y los intereses de los cientistas sociales por hacer un magíster en el extranjero. En efecto, desde una racionalidad estratégica, la realización de un magíster puede entenderse como una inversión particular difícilmente reducible a una instrumentalidad -principalmente monetaria y maximizadora- del interés. Esto no porque no exista tal instrumentalidad, pues de hecho efectivamente hay una orientación hacia un mejoramiento de las alternativas futuras; sino más bien porque el concepto por sí solo resulta muy estrecho para dar cuenta de la complejidad de las racionalidades y de las lógicas que se entrelazan en la definición del interés.

En relación con este hallazgo, hay aspectos que esta investigación deja abiertos y que sería interesante abordar posteriormente, como por ejemplo:

a) Profundizar en los mecanismos mediante los cuales las decisiones educacionales se relacionan con los procesos de individuación. Al respecto cabría preguntarse:

- ¿Qué capitales asociados al magíster se consideran relevantes para la individuación?

- ¿Cómo quieren ser estos estudiantes, es decir, qué tipo de individuación se cree que puede propiciar la realización de un magíster en el extranjero?

- ¿Qué significa crecimiento personal en estos contextos de estudio?

b) Indagar en las relaciones entre estas racionalidades y lógicas. Es dable pensar, en este caso, que haya complementariedades, contradicciones, jerarquías, etc.

c) Indagar en la posible existencia de otras racionalidades y lógicas intervinientes. 
d) Analizar estas racionalidades comparadamente entre tipos de profesionales, es decir: ¿la valoración de la experiencia es particular de los cientistas sociales?

Por otra parte, la información producida es valiosa en tanto permite realizar algunas consideraciones para la política pública de fomento al posgrado, específicamente para Becas Chile. Como no se trata de una evaluación de política pública, los aportes de este estudio se refieren a las potencialidades del programa, antes que al análisis de su funcionamiento y fallas.

Debido a los objetivos de Becas Chile y la conceptualización de la teoría del capital humano, se puede decir que esta política pública se apoya en la racionalidad estratégica de sus beneficiarios, en tanto incluye a la instrumentalidad que está en la base de la inversión en educación. Ahora, en consideración de la existencia de una racionalidad experiencial, ¿cómo esta interroga a Becas Chile?

Si se extrapolara el análisis poniendo a ambas racionalidades en una balanza, y se observara una preponderancia de la racionalidad estratégica frente a la experiencial, sería posible pensar que la política no requeriría cambios, o bien, que requeriría solo ajustes menores en su justificación y fundamentos. En cambio, si la racionalidad experiencial cobrara mayor preponderancia que la estratégica, se vuelven evidentes al menos dos potenciales caminos:

- Cuestionamiento de Becas Chile: ¿Es necesario fomentar los posgrados en el extranjero? La centralidad de la racionalidad experiencial no necesariamente evidenciaría una falla de la política pública, pero sí la interrogaría por su sentido: si el fin de Becas Chile es aumentar el capital humano avanzado del país, y no el crecimiento personal experiencial de las personas y su definición como individuos, puede cuestionarse la necesidad de que estos posgrados se hagan en el extranjero. De hecho, con los mismos recursos podrían apuntalarse los posgrados nacionales; así como también podría o no crearse otro tipo de política que se haga cargo del interés por lo que implica vivir en el extranjero en términos personales. 
- Reformulación de Becas Chile. Como segundo camino podría plantearse la necesidad de que Becas Chile se haga cargo de este componente experiencial, incluyéndolo en sus objetivos para así obtener de él provechos no solo a nivel individual, sino que también a nivel nacional. Ello requeriría de mecanismos para aprovechar las experiencias de los alumnos en el extranjero, a fin de transformarlas en aprendizajes para el país. Dicho en jerga antropológica, equivale a fortalecer la condición de etnógrafos de estos estudiantes que se van "a vivir la experiencia", propiciando por ejemplo que estos hagan "descripciones densas" (Geertz, 1992) acerca de cómo se está construyendo el conocimiento en su país de estudios; es decir, que se vuelvan investigadores de su experiencia. Estrategias como esta pueden ser de gran relevancia si consideramos que cada vez va a haber más posgraduados en Chile, muchos de ellos con estudios en el extranjero.

Por cierto, otra pregunta que surge es si pasará con la expansión de los posgrados lo mismo que con el pregrado, en tanto los títulos que, al saturarse, dejan de representar una distinción. Esto es algo que aquí se dejará abierto.

Con todo, más que dilucidar sus fallas, los hallazgos ponen en tensión la consistencia entre los objetivos del programa Becas Chile y el carácter internacional de las becas que financia. En este sentido, contribuyen a desnaturalizar la necesidad de esta política, abriendo caminos que pueden contribuir a socavarla, o bien, a enriquecerla.

Los resultados, además, evidencian la urgencia de ir, de manera constante, a los sujetos implicados en esta política, no solo para evaluar sus efectos, sino también -y primordialmente- para transformarla. En efecto, puede ser de gran utilidad fortalecer los mecanismos de autoobservación de las instituciones a cargo, en este caso de Conicyt. Esto equivale a generar un sistema de estudio permanente en torno a los resultados y consecuencias individuales y sociales de la política, de modo que esta se vaya modulando en concordancia con la realidad a la que apunta. 


\section{Referencias}

Atria, R. (2004). Estructura ocupacional, estructura social y clases sociales. Santiago: División de Desarrollo Social, CEPAL.

Becas Chile (2009). ¿Qué es Becas Chile? Objetivos. Recuperado el día 20 de febrero de 2014 desde http://www.becaschile.cl/index3.php?id_ contenido=20988\&id_portal=60\&id_seccion $=4276$

Becas Chile (2012). Estadísticas concurso magíster. Recuperado el día 20 de febrero de 2014 desde http://www.becaschile.cl/index2.php?id_ seccion=4003\&id_portal=60\&id_contenido=17946

Becas Chile (2013). Estadísticas generales (todos los concursos en conjunto). Recuperado el día 20 de febrero de 2014 desde http://www.becaschile. cl/index2.php?id_portal=60\&id_seccion=3536\&id_contenido=15472

Becker, G. (1983). El capital humano. Madrid: Alianza Editorial.

Becker, G. (1990). The economic approach to human behavior. Estados Unidos: Universidad de Chicago. Recuperado el día 20 de febrero de 2014 desde http://books.google.cl/books?hl=es\&lr=\&id=qQAZnc-mMSoC\&oi=fn $\mathrm{d} \& \mathrm{pg}=\mathrm{PAl} \& \mathrm{dq}=$ becker+the+economic + approach $\&$ ots=2FRjlvMNSj\& sig=qbmnCcBKeVrOAu_SmymlGEu-t8I\#v=snippet\&q=materials\%20 goods $\& f=$ false

Bourdieu, P. (1991). El sentido práctico. Madrid: Ed. Taurus Humanidades.

Bourdieu, P. (1997). Razones prácticas: sobre la teoría de la acción. Barcelona: Editorial Anagrama.

Bourdieu, P. (2003). Las estructuras sociales de la economía. Barcelona: Editorial Anagrama.

Brunner, J.J. y Uribe, D. (2007). Mercados universitarios: el nuevo escenario de la educación superior. Santiago: Ediciones Universidad Diego Portales.

CEPAL (2000). La brecha de la equidad: una segunda evaluación. Segunda conferencia regional de seguimiento de la cumbre mundial sobre desarrollo social, 15 al 17 de mayo de 2000, Santiago de Chile. Recuperado el 20 de febrero de 2014 desde: www.eclac.org/ publicaciones/xml/3/4303/lcg2096e.pdf

CNAP (2007). CNAP 1999-2007: el modelo chileno de acreditación de la educación superior. Santiago: Ministerio de Educación.

Collins, R. (1989). La sociedad credencialista: sociología histórica de la educación y de la estratificación. Madrid: Akal.

Crompton, R. (1994). Clase y estratificación. Una introducción a los debates actuales. Madrid: Editorial Tecnos. 
CSE (2011). Índice digital 2011. Recuperado el día 20 de marzo de 2014 desde http://www.cned.cl/public/Secciones/SeccionIndicesEstadisticas/ indices_estadisticas_sistema.aspx.

Destinobles, A. (2006). El capital humano en las teorías del crecimiento económico. México: Universidad Autónoma de Chihuahua, Escuela de Economía Internacional. Recuperado el día 20 de marzo de 2014 desde http:// www.eumed.net/libros/2006a/agd/lc.htm.

Didriksson, A. (2008). Contexto global y regional de la educación superior en América Latina y el Caribe. Conferencia Regional de Educación Superior, 4 al 6 de junio de 2008, Cartagena de Indias, Colombia.

Emol (2008). Discurso presidencial 2008. Contigo mejor país. Contigo vamos por más. Recuperado el día 20 de marzo de 2014 desde http://www. emol.com/noticias/documentos/pdfs/discursopresidencial_21dema yo2008.pdf

Franco, R. (2002). La educación y la segunda generación de reformas en América Latina. Recuperado el día 20 de marzo de 2014 desde http:// www.rieoei.org/rie30a05.htm

Geertz, C. (1992). Descripción densa: hacia una teoría interpretativa de la cultura. Recuperado el día 20 de febrero de 2014 desde http://www. cholonautas.edu.pe/modulo/upload/geer.pdf

Godelier, M. (1966). Racionalidad e irracionalidad en la economía. México: Siglo XXI editores.

Lévi-Strauss, C. (1964). El pensamiento salvaje. México: Fondo de Cultura Económica.

Marginson, S. (2008). Global field and global imaging: Bourdieu and worldwide higher education. British Journal of Sociology of Education, 29(3), 303-315. Doi: 10.1080/01425690801966386. Recuperado el día 20 de febrero de 2014 desde http://www.cshe.unimelb.edu.au/ people/marginson_docs/BJSE\%202008.pdf

Márquez, M. y Heyl, V. (2010). El sistema bicentenario Becas Chile. Una evaluación de la política pública de formación de capital humano avanzado 2006-2010. Santiago: Conicyt. Recuperado el día 20 de febrero de 2014 desde http://www.sociedadpoliticaspublicas.cl/ archivos/BLOQUE1/Educacion_superior_y_Eduacion_Continual El_Sistema_Bicentenario_Becas_Chile.pdf

Martuccelli, D. (2007). Cambio de rumbo. La sociedad a escala del individuo. Santiago: LOM Ediciones.

Mauss, M. (1971). Ensayo sobre los dones: razón y forma del cambio en las sociedades primitivas. Madrid: Editorial Tecnos. 
Mideplan, División Social (1992, 1994, 1996, 1998, 2000, 2003, 2006, 2009 ). Encuesta CASEN. Recuperado el día 20 de febrero de 2014 desde www.mideplan.cl/casen

Mineduc (2009). Decreto Supremo No 664. Recuperado el 20 de febrero de 2014 desde http://www.mineduc.cl/usuarios/bchile/doc/201206261712260. DECRETO\%20664.pdf

Mincer, J. (1958). Investment in human capital and personal income distribution. The Journal of Political Economy, 66(4), 281-302. Doi:10.1086/258055

Montuschi, L. (2006). El libre albedrío y los conceptos de racionalidad y de elección racional en la ciencia económica. Revista de Economía y Estadística (2), 27-58.

OCDE-Banco Mundial (2011). Revisión de politicas nacionales de educación. Programa Becas Chile. Santiago: Ministerio de Hacienda de Chile.

Parsons, T. (1959). The School Class as a Social System: some of its Functions in American Society. Harvard Educational Review, 29, 297-318.

Passeron, J.C. (1983). La inflación de los títulos escolares en el mercado del trabajo y el mercado de los bienes simbólicos. Revista Educación y Sociedad, 1, 5-27.

Patrón, R. (2008). La educación como inversión y como actividad productiva formadora de recursos. Principales aspectos teóricos. Notas docentes No 21. Uruguay: Universidad de la República.

Polanyi, K. (1989). La gran transformación. Madrid: La Piqueta.

Rodríguez-Sickert, C. (2009). Homo economicus. Recuperado el día 20 de febrero de 2014 desde http://www.complejidadsocial.cl/wp-content/ uploads/2012/05/Homo-economicus-Handbook-E.Elgar_.pdf

Rodríguez, C. y Vio, C. (2003). Necesidad de posgraduados en Chile. En Estudios de posgrado. Perspectivas y desafios (pp. 21-39). Santiago: Publicaciones CSE.

Sahlins, M. (1983). Economía de la Edad de Piedra. Madrid: Akal.

Schultz, T. (1960). Capital formation by education. Recuperado el día 20 de febrero de 2014 desde http://ideas.repec.org/a/ucp/jpolec/ v68y1960p571.html (Doi: 10.1086/258393)

Spencer, E. (2003). Políticas para el desarrollo de un sistema nacional de cuarto nivel. En Estudios de Posgrado. Perspectivas y desafíos (pp. 41-46). Santiago: Publicaciones CSE. 
Statcom Estadística (2007). Evaluación en profundidad de los programas de becas de posgrados. DIPRES, Santiago. Recuperado el día 20 de febrero de 2014 desde http://geminis.dipres.cl/VirLib/fr_consultaEvaluacion.htm

Tudela, P. (2006). El rol del análisis en la investigación: aspectos básicos en el análisis del material etnográfico. Santiago: Universidad de Chile, Facultad de Ciencias Sociales.

Universia (2007). Entregan becas Presidente de la República a beneficiarios del proceso 2007. Recuperado el día 31 de marzo de 2014 desde: http://becas.universia.net/CL/noticia/444/entregan-becas-presidenterepublica-beneficiarios-proceso-2007.html

Recibido: 31/03/2014

Aceptado: 26/05/2014 